\title{
Resilience and self-harm among left-behind children in Yunnan, China: a community- based survey
}

\author{
Xin Tian ${ }^{1,2}$, Wei Chang ${ }^{1}$, Qiong Meng ${ }^{1}$, Ying Chen ${ }^{1}$, Zhen Yu', Limei He ${ }^{1}$ and Yuanyuan Xiao ${ }^{1,3^{*}}$ (D)
}

\begin{abstract}
Background: Self-harm (SH) behaviors are established risk factors of suicide, however, in Chinese left-behind children (LBC), SH remains severely under-discussed. In this study, we aimed to investigate SH and explore its association between resilience in a large group of LBC.

Methods: A community-based cross-sectional study of 2898 LBC was conducted in Yunnan province, China. Information was collected by self-reporting questionnaires. Descriptive analysis was used to depict and compare characteristics of the subjects. Univariate and multivariate logistic regression models were applied to estimate the associations between resilience and SH prevalence, SH severity, SH repetition.

Results: The prevalence of SH in LBC was $48.8 \%$ (95\%Cl: 47.0-49.7\%). Compared to LBC with lower level of resilience, a higher level of resilience was related to 0.40 folds of SH odds ( $95 \% \mathrm{Cl}$ : $0.34-0.48$ ). Besides, among all dimensions of resilience, every 1 average score increase of emotion regulation and family support were associated with 0.13 (95\% Cl: $0.04-0.37$ ) and 0.14 (95\%Cl: 0.04-0.47) folds of odds in severer SH, respectively; one unit increase in the average score of emotion regulation was related to an OR of 0.23 (95\%Cl: 0.07-0.71) for repeated SH.

Conclusions: Psychological resilience presented protective effect on SH in LBC, especially the dimensions of emotion regulation and family support. Intervention measures focusing on enhancing emotion regulation ability and building parent-child connection could be considered in order to reduce SH and suicidal risk in LBC.
\end{abstract}

Keywords: Left-behind children, Self-harm, Resilience, Emotion regulation, Family support

\section{Background}

With the widening rural-urban split exacerbated by economical prosperity and urbanization in the last four decades in China, increasing flow of rural labors into big cities for job opportunities has generated an expanding population known as the left-behind children (LBC), which indicates to children been left at home by one or both of their migrated parents [1,2]. According to the released data in 2013 by All China Women's Federation, the number of LBC had climbed to a staggering 61 million, accounted for $37.7 \%$ of the rural children and $21.9 \%$ of the total Chinese children $[3,4]$. The overwhelmingly majority

\footnotetext{
*Correspondence: 33225647@qq.com

'Department of Epidemiology and Health Statistics, School of Public Health, Kunming Medical University, Kunming 650500, Yunnan, China

${ }^{3}$ Department of Chronic Disease Epidemiology, Yale School of Public Health Yale University, New Haven, CT, USA

Full list of author information is available at the end of the article
}

of $\mathrm{LBC}$ are from inland destitute areas, which made LBC a socioeconomically deprived population. Moreover, compared to non-left-behind children (NLBC), the long separation from parents will put LBC into an even more vulnerable situation. Therefore, in recent years, concerns regarding to the health status of $\mathrm{LBC}$ have been accumulated [5-7].

Self-harm $(\mathrm{SH})$ refers to the behaviors that individual adopted to hurt oneself regardless of the intention [8]. It can occur at any age but reportedly more common in pubertal phase [9]. In China, a multi-center study based on a sample of 11,880 adolescents reported a SH prevalence of $30.3 \%$ [10]. Individual, familial, and social environmental determinants may collectively contribute to the increased $\mathrm{SH}$ in adolescents, and among them, it has been suggested that living in structurally or functionally abnormal family was associated with increased risk of 
$\mathrm{SH}$ [11]. Therefore, it is reasonable to suspect that, $\mathrm{SH}$ incidence of LBC will be significantly higher than NLBC. In fact, two previous cross-sectional studies published in Chinese have preliminarily investigated this issue with supportive evidence [12, 13]. Existing studies highlight the importance of $\mathrm{SH}$ in suicide, as it has been estimated that approximately $50-60 \%$ of suicidal deaths were coupled with a history of $\mathrm{SH}$, and $\mathrm{SH}$ adolescents reported threefold risk of suicidal ideation (SI) compared with their non-SH counterparts $[14,15]$. A recently published study found that, the one-year prevalence of suicide attempt in Chinese LBC was 3.75\%, significantly higher than which in NLBC (2.86\%) [16]. Therefore, it is of great importance to investigate $\mathrm{SH}$ and its influencing factors in Chinese LBC, so as to effectively reduce future suicidal risk in this vulnerable population. However, up till now, this topic remains severely under-discussed.

In the field of psychology, resilience is described as a competence of individual to successfully pull through adversity or trauma [17]. A newly published meta-analysis revealed a lower resilience level of Chinese LBC than NLBC [18]. Western literature reported that resilience was significantly associated with $\mathrm{SH}$ : $\mathrm{SH}$ individuals were generally observed lower level of resilience [19]. With this regard, resilience building might be a promising method to alleviate SH frequency and severity among LBC. It is also practical, for empirical evidence has suggested that individual resilience can be drastically reinforced by psychological or psychosocial intervention measures [20]. Nevertheless, this possible protective role of resilience in $\mathrm{SH}$ has never been explored in Chinese LBC.

Aiming at the deficiencies in current literature mentioned above, we intended to intensively analyze the association between resilience and $\mathrm{SH}$ in a large communitybased sample of Chinese LBC. We hypothesized that, resilience is inversely associated with $\mathrm{SH}$ prevalence, $\mathrm{SH}$ severity, and $\mathrm{SH}$ repetition.

\section{Methods}

\section{Study population and procedure}

After the Ethics Review Board of Kunming Medical University approved, we performed a cross-sectional survey in Guangnan, a county affiliated to Wenshan Zhuang and Miao Autonomous Prefecture, home to the largest number of LBC among ethnical minority prefectures in Yunnan province, China. The survey was performed from June 26 to July 6, 2018. A one-stage random cluster sampling was applied: 1) Three townships were randomly chosen from a total of 18 rural townships in Guangnan; 2) All eligible LBC within the chosen townships were preliminarily included. We then used the following inclusion criteria to further screen study subjects: 1) One or both of their parents had been migrated for at least 6 months in the most recent year; 2) Aged between
10 and 17 years; 3) Resided in survey sites for at least six months per year. Children or adolescents were further excluded if they were: 1) Illiterate; 2) Delirious or suffering from severe psychiatric disorder with incompetence in expression; 3) Hearing or speech dysfunction; 4) Critically ill or under inconvenient conditions; 5) Refuse to participate.

Sample size. We chose a conservative estimation of $20 \%$ for $\mathrm{SH}$ prevalence based on published literature. The acceptable error rate and statistical significance level were set as $3 \%$ and 0.05 , respectively. By using the formula for simple random sampling $\left(N=\frac{Z_{2 / \alpha}^{2} \pi(1-\pi)}{\delta^{2}}\right)$, we calculated a preliminary sample size of 712 . Given the possible advent of refusal, an adjusted sample size was obtained on the premise of $80 \%$ effective response rate $(N=712 / 0.8=890)$. Considering that the sampling error of cluster sampling is inevitably bigger that simple random sampling, a design effect of 2 was applied to further adjust the required sample size, therefore, the final calculated sample size was $N=890 \times 2=1780$.

Measurements. Face-to-face interview was applied to collect information from study subjects by paper-andpencil approach. Interviewers were qualified senior class undergraduates or graduates majoring in clinical medicine or public health from Kunming medical university. Before survey, all interviewers accepted a one-day intensive training and passed the subsequent examination. Prior to interview, a written consent was obtained from either the parent or legal guardian or teacher of the participant, as appropriate to the specific situation. The instrument we used contains 5 parts, which measure general characteristics, SH behaviors, psychological resilience, depressive symptoms (measured by the Chinese Version of Children's Depression Inventory), and SI (measured by the Chinese Version of Beck Scale for Suicide Ideation) of LBC, respectively. The current study only used variables from the first three parts.

Self-harm. Self-harm was measured by using the Modified version of Adolescents Self-Harm Scale (MASHS) developed by Feng [21]. MASHS measures both the lifetime frequency and severity of the 18 most commonly reported $\mathrm{SH}$ behaviors among Chinese adolescents. Frequency of SH was measured by scaled options: never, once, two to four times, five times and above. SH severity was divided into 5 levels: non-observable injury, slight injury (observable but no need to treat), medium injury (requires simple treatment, no need to visit medical facilities), severe injury (requires treatment in medical facilities, no need to be hospitalized), critical injury (requires urgent treatment in emergency room and the subsequent hospitalization). The Cronbach's $\alpha$ of MASHS in this sample was 0.75 , indicates an acceptable level of internal consistency. 
Resilience. We adopted the Resilience Scale for Chinese Adolescents (RSCA) designed by $\mathrm{Hu}$ and Gan [22]. The respondents were expected to answer the degree of pertinence of the 27 items based on their own situation. Answers were rated by a five-point Likert scale: totally disagree ( 1 point), disagree (2 points), not sure (3 points), agree ( 4 points), and totally agree (5 points). The combined score of RSCA ranges from 27 to 135 , with a higher sum indicates a better resilience level. The Cronbach's $\alpha$ of RSCA in our study was 0.77 . Based on exploratory factor analysis of the designer, the 27 items of RSCA can be grouped into 5 dimensions: goal concentration, emotion regulation, positive perception, family support, and interpersonal assistance.

\section{Statistical analysis}

Descriptive statistics were used to depict general characteristics of LBC. The categorical variables were presented with frequencies and proportions while the continuous variables were described by means and standard deviations (SDs). We dichotomized study subjects into two subgroups based on the presence of any SH behavior collected by MASHS. Univariate logistic regression model with a less strict significance level of $p<$ 0.1 was adopted to screen for possible associated factors of SH. Multivariate logistic regression model was latter applied to estimate the adjusted associations between identified factors and SH. Finally, we performed a subgroup analysis only in self-harmed LBC: they were dichotomized by using $\mathrm{SH}$ severity (the presence of medium to severe $\mathrm{SH}$ ) and $\mathrm{SH}$ repetition (the presence of more than once $\mathrm{SH}$ ) separately, associated factors of $\mathrm{SH}$ severity and repetition were also analyzed by using univariate and multivariate logistic regression models. All statistical analyses were performed using SPSS 21.0 (SPSS Inc., USA). Statistical significance threshold of $p$ value was set at no more than 0.05 , two-tailed.

\section{Results}

\section{General characteristics of participants}

By applying inclusion and exclusion criteria mentioned above, altogether 3011 LBC were initially determined. Among them, 28 declined to participate, 10 were latter confirmed over-aged ( $\geq 18$ years), and 75 were further deleted because of missing information in critical items, which left 2898 subjects for further analysis. The valid response rate was $96.6 \%$. The major characteristics of study subjects were listed in Table 1: the mean age was 14.4 years with a SD of 1.81. Middle school students took the predominant majority $(N=1622,55.97 \%)$. A total of $1413 \mathrm{LBC}$ reported engaged in at least one episode of $\mathrm{SH}$, with a lifetime prevalence of $48.80 \%$ (95\% CI: 47.00-49.70\%); slight injury was the commonest type of reported $\mathrm{SH}(N=1037 ; 73.39 \%)$. In self-harmed LBC,
Table 1 Descriptive statistics of 2898 LBC analyzed

\begin{tabular}{|c|c|c|}
\hline Variable & $N(\%)$ & Mean (SD) \\
\hline Age & & $14.46 \pm 1.81$ \\
\hline \multicolumn{3}{|l|}{ Gender } \\
\hline Male & $1504(51.90)$ & \\
\hline Female & $1394(48.10)$ & \\
\hline \multicolumn{3}{|l|}{ Ethnicity } \\
\hline Han & $532(18.36)$ & \\
\hline Zhuang & $1624(56.04)$ & \\
\hline Miao & 605 (20.88) & \\
\hline Other & $137(4.72)$ & \\
\hline \multicolumn{3}{|l|}{ Grade } \\
\hline Primary school & $748(25.81)$ & \\
\hline Middle school & $1622(55.97)$ & \\
\hline High school & $528(18.22)$ & \\
\hline \multicolumn{3}{|l|}{ Education of father } \\
\hline Illiterate or semi- illiterate & $1055(36.40)$ & \\
\hline Primary school & $1130(38.99)$ & \\
\hline Middle school or above & $512(17.67)$ & \\
\hline Missing & $201(6.94)$ & \\
\hline \multicolumn{3}{|l|}{ Education of mother } \\
\hline Illiterate or semi-illiterate & $1830(63.15)$ & \\
\hline Primary school & $644(22.22)$ & \\
\hline Middle school and above & $193(6.66)$ & \\
\hline Missing & $231(7.97)$ & \\
\hline Age of father & & $39.45 \pm 5.44$ \\
\hline Age of mother & & $37.78 \pm 5.16$ \\
\hline \multicolumn{3}{|l|}{ Self-harm behavior } \\
\hline Yes & $1413(48.76)$ & \\
\hline No & $1485(51.24)$ & \\
\hline \multicolumn{3}{|l|}{ Self-harm severity } \\
\hline Slight & 1037(73.39) & \\
\hline Medium or above & $376(26.61)$ & \\
\hline \multicolumn{3}{|l|}{ Self-harm repetition } \\
\hline Yes & $1017(71.97)$ & \\
\hline No & $396(28.03)$ & \\
\hline Resilience score & & $94.42 \pm 12.85$ \\
\hline Goal concentration & & $3.77 \pm 0.73$ \\
\hline Interpersonal assistance & & $3.42 \pm 0.85$ \\
\hline Emotion regulation & & $3.15 \pm 0.78$ \\
\hline Positive perception & & $3.71 \pm 0.71$ \\
\hline Family support & & $3.55 \pm 0.68$ \\
\hline
\end{tabular}

1017 (71.97\%) reported repetitive SH. The overall score of psychological resilience and its SD were 94.42 and 12.85 , respectively. Based on the median RSCA score, subjects were dichotomized as low resilience group 
$(\mathrm{RSCA} \leq 94)$ and high resilience group (RSCA $>94)$. The average scores of the five specific dimensions of resilience varied, ranging from $3.15 \pm 0.78$ (emotion regulation) to $3.77 \pm 0.73$ (goal concentration).

\section{Associated factors of SH}

As shown in Table 2, variables with $p$ values less than 0.10 in univariate analysis were: gender, grade, father's age, mother's age, father's education level, and resilience. After multivariate adjustment, the association between resilience and $\mathrm{SH}$ stayed statistically prominent: compared to LBC with lower resilience level (RSCA<94), a higher resilience level $(\mathrm{RSCA} \geq 40)$ was related to 0.40 folds of SH odds (95\%CI: 0.34-0.48). We fitted multivariate logistic regression models which included the 5 specific dimensions of RSCA instead of the combined scores separately, and the results revealed that: except for positive perception, all the other four dimensions were significantly inversely associated with SH. Among the four dimensions, emotion regulation showed the strongest association: every 1 average score increase was associated with an odds ratio (OR) of 0.03 (95\%CI: 0.01-0.06) in SH.

\section{Resilience with SH severity and repetition}

We further discussed the possible influence of dimensions of resilience on SH severity and repetition in self-

Table 2 Univariate and multivariate analyses for self-harm behaviors in all left-behind children

\begin{tabular}{|c|c|c|c|c|c|c|}
\hline \multirow[t]{2}{*}{ Variable } & \multicolumn{2}{|l|}{ Univariate model } & \multicolumn{2}{|c|}{ Multivariate model 1} & \multicolumn{2}{|c|}{ Multivariate model 2} \\
\hline & $\mathrm{OR}(95 \% \mathrm{Cl})$ & $\mathrm{OR}(95 \% \mathrm{Cl})$ & $p$ value & & $\mathrm{OR}(95 \% \mathrm{Cl})$ & $p$ value \\
\hline \multicolumn{7}{|l|}{ Gender } \\
\hline Female & Ref & & & & & \\
\hline Male & $1.25(1.08-1.44)$ & $<0.01$ & $0.78(0.66-0.92)$ & $<0.01$ & $0.72(0.61-0.86)$ & $<0.01$ \\
\hline \multicolumn{7}{|l|}{ Ethnicity } \\
\hline Han & Ref & & & & & \\
\hline Zhuang & $1.08(0.89-1.31)$ & 0.45 & - & - & - & - \\
\hline Miao & $1.10(0.87-1.39)$ & 0.44 & - & - & - & - \\
\hline Other & $0.87(0.59-1.26)$ & 0.46 & - & - & - & - \\
\hline \multicolumn{7}{|l|}{ Grade } \\
\hline Primary School & Ref & & & & & \\
\hline Middle School & 1.65 (1.39-1.97) & $<0.01$ & $1.66(1.35-2.04)$ & $<0.01$ & $1.56(1.27-1.93)$ & $<0.01$ \\
\hline High school & $0.96(0.76-1.20)$ & 0.70 & $0.99(0.77-1.29)$ & 0.96 & $0.88(0.67-1.15)$ & 0.36 \\
\hline Father's age (+ 1 year) & $1.02(1.01-1.03)$ & 0.01 & $1.02(0.99-1.04)$ & 0.22 & $1.02(0.99-1.05)$ & 0.20 \\
\hline Mother's age (+ 1 year) & $1.02(1.00-1.03)$ & 0.04 & $0.10(0.97-1.03)$ & 0.89 & $0.10(0.97-1.02)$ & 0.72 \\
\hline \multicolumn{7}{|l|}{ Father's education level } \\
\hline Illiterate/semi-illiterate & Ref & & & & & \\
\hline Primary School & $0.84(0.71-1.00)$ & 0.05 & $0.82(0.68-0.98)$ & 0.03 & $0.80(0.66-0.96)$ & 0.02 \\
\hline Middle School or above & $0.77(0.63-0.96)$ & 0.02 & $0.80(0.64-1.01)$ & 0.07 & $0.78(0.62-0.99)$ & 0.04 \\
\hline \multicolumn{7}{|l|}{ Mother's education level } \\
\hline Illiterate/semi-illiterate & Ref & & & & & \\
\hline Primary School & $0.91(0.76-1.09)$ & 0.32 & - & - & - & - \\
\hline Middle School or above & $0.87(0.65-1.17)$ & 0.36 & - & - & - & - \\
\hline \multicolumn{7}{|l|}{ Resilience } \\
\hline $\operatorname{RSCA}<94.0$ & Ref & & & & & \\
\hline $\mathrm{RSCA} \geq 94.0$ & $0.42(0.36-0.48)$ & $<0.01$ & $0.40(0.34-0.48)$ & $<0.01$ & - & - \\
\hline \multicolumn{7}{|l|}{ Resilience dimensions } \\
\hline Emotion regulation (+ 1 average score) & & & - & - & $0.03(0.01-0.06)$ & $<0.01$ \\
\hline Goal concentration (+ 1 average score) & & & - & - & $0.26(0.10-0.69)$ & 0.01 \\
\hline Interpersonal assistance (+ 1 average score) & & & - & - & $0.17(0.08-0.36)$ & $<0.01$ \\
\hline Positive perception (+ 1 average score) & & & - & - & $1.83(0.69-4.84)$ & 0.23 \\
\hline Family support (+ 1 average score) & & & - & - & $0.26(0.09-0.70)$ & 0.01 \\
\hline
\end{tabular}


harmed LBC. By taking SH severity as the dependent, after adjusted for other possible influencing covariates, only emotion regulation and family support were statistically inversely associated with SH severity: every 1 average score increase of the two dimensions were associated with 0.13 (95\%CI: $0.04-0.37)$ and 0.14 (95\%CI: 0.04-0.47) folds of odds in medium or above injuries (Fig. 1, panel a).

By using the same analytical strategy, for 5 dimensions of resilience, only emotion regulation was prominent factor of SH repetition: one unit increase in the average score was related to an OR of 0.23 (95\%CI: 0.07-0.71) for repeated SH (Fig. 1, panel b).

\section{Discussion}

In the current study, we thoroughly discussed the association between $\mathrm{SH}$ and resilience in a large group of Chinese LBC, an interesting and important topic that has never been adequately addressed. Based on analytical results, we found that, $\mathrm{SH}$ is an urgent challenge in $\mathrm{LBC}$, as a hefty $48.8 \%$ of self-reported $\mathrm{SH}$ prevalence has been revealed, much higher than which in other regions of China [23-25]. Besides, much in accordance with our anticipation, although the strengths and patterns of the associations varied, resilience was in general inversely associated with prevalence, severity, and repetition of $\mathrm{SH}$ behaviors.

The positive association between resilience and $\mathrm{SH}$ prevalence coincides with prior findings that higher levels of resilience potentially favored $\mathrm{SH}$ protection amid Chinese adolescents [26, 27], and non-SH adolescents tend to be more resilient than self-injurers [19]. The adolescent resilience model proposed by Haase [28] reckoned that, by gaining resources from individual, family and society, resilience renders people more prone to obtain resilience-related self-esteem, confidence and life satisfaction. The integrated model of self-injury by Nock [29] emphasized the combined effect of peers, family, schools and society in triggering the advent of self-injury. Therefore, one possible explanation could be that, more resilient individuals enjoy an advantage in multiple resources, which may predispose them to a lowered risk of SH. Additionally, resilience was believed to be a moderator between negative life events and mental health problems [30], and the latter were established risk factors of $\mathrm{SH}$ behaviors [31]. With this regard, LBC presented with higher resilience may less likely to be plagued by mental health problems like depression, which in turn, curtails the presence of $\mathrm{SH}$.

Further analysis revealed that, among the five dimensions of resilience, emotion regulation was consistently associated with $\mathrm{SH}$ prevalence, severity, and repetition. Among general adolescents, emotion regulation was deemed plays a crucial role in depression and anxiety, as it

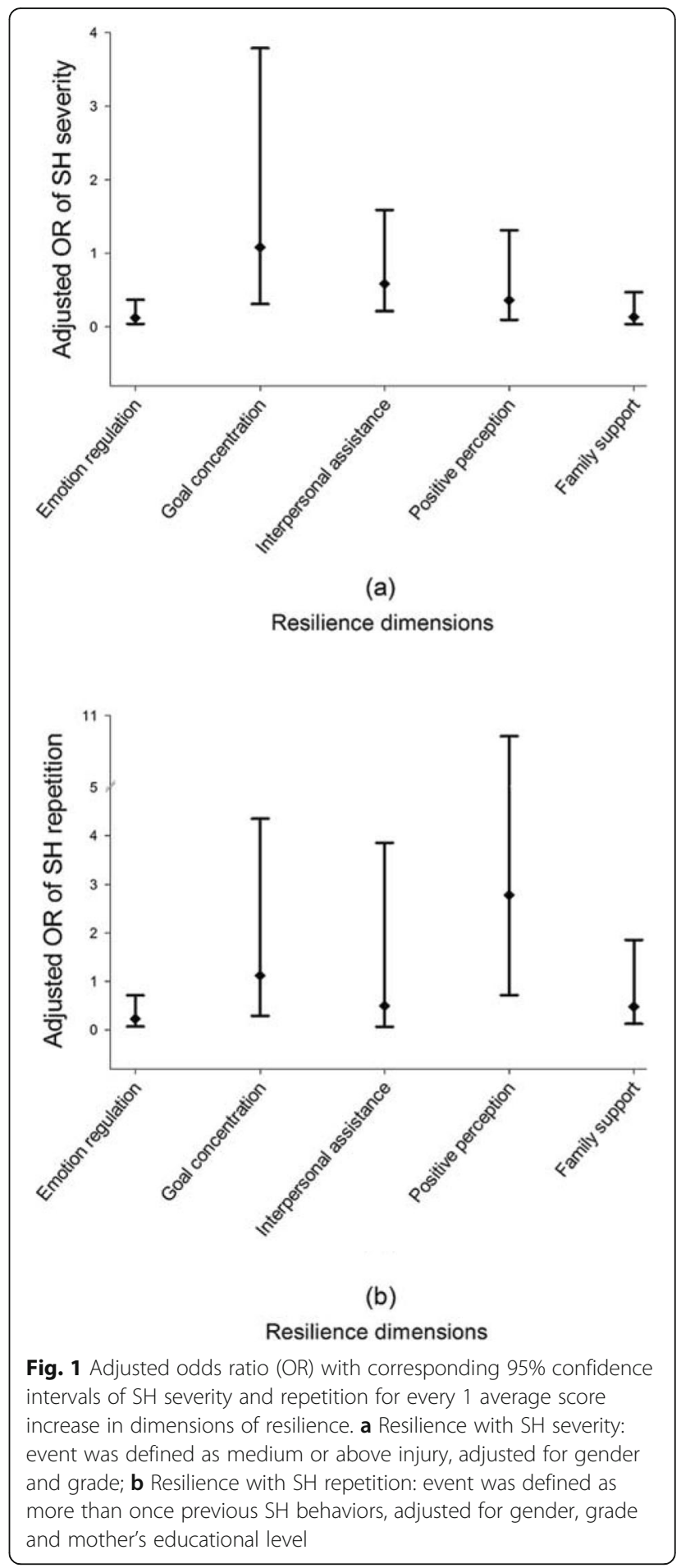

has been suggested that the unsatisfactory emotion regulation was related to reduced capability in dealing with emotional challenges [32, 33]. In view of the intimate relationship between affective disorders, typically depression and anxiety, and $\mathrm{SH}$ behaviors in youngsters [34, 35], one possible explanation could be that, good emotion regulation dissuades self-mutilations engagement. The 
affect regulation model explains that, self-injury can occur with the purpose of keeping strong negative feelings regulatory [36]. Hence, the lack of emotion regulation ability may relate to susceptibility of immersing in negative mood, and consequently, the adoption of SH behaviors.

Another important finding was that, children with less perceived family support had higher incidence and severer $\mathrm{SH}$ behaviors, which was in agreement with several previous studies [37-39]. We found no such association existed in $\mathrm{SH}$ repetition, which was discrepant with the findings of Wang et al. [7]. However, different measurements of family support were used. Less intimacy and lower communication frequency between LBC and their migrated parents can increase the likelihood of encountering more loneliness and depression [40, 41]. In LBC, it has been reported that parent-child attachment negatively related to loneliness [40], and longitudinal evidence indicated that this attachment ameliorated the depression of LBC subsequently [42]. Therefore, it is reasonable to hypothesize that family support confers protective effect against $\mathrm{SH}$ in LBC by reducing loneliness and depression. Nevertheless, this assumption should be further validated.

Our major findings are of important secular significance. First and foremost, the striking prevalence of $\mathrm{SH}$ in LBC highlights the importance and urgency of SH intervention for the government and communities to effectively prevent or reduce such hazardous behavior in this mentally disadvantageous population Besides, the role of psychological resilience should be stressed. This is also a feasible intervention direction, as existing evidence suggests that resilience could be effectively enhanced through various kinds of group-based counseling measures among teens $[20,43]$.

As aforementioned, when dissected, emotion regulation was the strongest indicator of $\mathrm{SH}$ severity and repetition among all 5 dimensions of resilience. One study published in Chinese has already disclosed promising results: intervention on emotion regulation was associated with reduced level of SH frequency in a small group of LBC chosen from a single rural middle school [44]. The approaches the authors adopted, such as the correction of erroneous perceptions on $\mathrm{SH}$, challenging unreasonable faith, and relaxation training, are generally practicable in school or community based resilience intervention programs for LBC.

Moreover, parent-child relationship should also be strengthened. Timely and adequate face-to-face communication is the best way to cultivate intimacy between children and their parents. However, it is unrealistic for LBC. Therefore, other possible substitutes should be considered. Perhaps a higher contact frequency via multiple means of modern communication can be an expedient but ideal choice, such as telephone, instant text message, audio or video chat, etcetera, as some positive conclusions have been reached by empirical studies $[45,46]$. Among all available and affordable choices, which communication method outperforms the rest will be an interesting topic to be discussed.

The most important insight of our findings is that, not only the multidimensional nature of psychological resilience should be considered, the characteristics of $\mathrm{SH}$ is of identical importance to think about. Intervention measures that focus on different dimensions of resilience in combination of severity and repetition of $\mathrm{SH}$ may be the most effective: for $\mathrm{LBC}$ engaged in repeated $\mathrm{SH}$, emotion regulation ability building should be stressed, whereas for LBC adopted severer SH behaviors, measures on parent-child relationship improvement could be simultaneously involved.

In this community-based study, we exhaustively discussed the association between resilience and $\mathrm{SH}$ with meaningful results. The reliability and validity of our findings can be substantially consolidated by representative sampling strategy and expanded sample size. Nevertheless, several limitations should be noticed. First, this is a cross-sectional study, which means that the causal inference cannot be reached. Second, information bias could exist for all information was collected by selfreporting. Finally, the study subjects were chosen from a single location in west China, thus discretion is needed in extrapolating the status of the whole Chinese LBC population based on our observations. Future studies with representative large sample and prospective design are warranted to further corroborate our findings. Besides, the underlying mechanism behind resilience-SH association should also be investigated.

\section{Conclusions}

$\mathrm{SH}$ prevalence is high among Chinese $\mathrm{LBC}$ and resilience may serve as a protective factor. Among the five dimensions of resilience, emotion regulation was the strongest associated factor of $\mathrm{SH}$, and it also consistently correlated with $\mathrm{SH}$ severity and repetition. Besides, family support played a significant role in SH severity of LBC. These findings highlight the urgency of intervention aims at reinforcing emotion regulation and strengthening parent-child intimacy among this vulnerable population.

\section{Abbreviations \\ LBC: left-behind children; MASHS: the Modified version of Adolescents Self- Harm Scale; NLBC: non-left-behind children; RSCA: the Resilience Scale for Chinese Adolescents; SD: standard deviation; SH: Self-harm; SI: suicidal ideation}

\section{Acknowledgments}

Not applicable.

\section{Authors' contributions}

$Y X$ and $X T$ designed the study. YX, WC, QM, and YC carried out data collection, $X T$ and $Y X$ performed data analysis, $X T$ prepared the draft 
manuscript, YX critically revised the manuscript, YC, ZY and LH contributed to study design and editing of the manuscript. All authors have read and approved the manuscript.

\section{Funding}

The study is supported by Yunnan Applied Basic Research Projects-Kunming Medical University Union Foundation (No. 2018FE001(-132)), National Natural Science Foundation of China (No. 81703324), China Scholarship Council (No. 201808535098). The funders played no roles in study design, collection, analysis and interpretation of data and in writing the manuscript.

\section{Availability of data and materials}

The datasets analyzed during the current study are available from the corresponding author on reasonable request.

\section{Ethics approval and consent to participate}

This study was approved by the Ethics Review Board of Kunming Medical University. In addition, a written consent was obtained from either the parent or legal guardian. For boarding students, upon the approval of the ethics review board, written consents were obtained from teachers. All participated students aged 16 or above also provided written consents to participate by themselves.

\section{Consent for publication}

Not applicable.

\section{Competing interests}

The authors declare that they have no competing interests.

\section{Author details}

'Department of Epidemiology and Health Statistics, School of Public Health, Kunming Medical University, Kunming 650500, Yunnan, China. ${ }^{2}$ The First Affiliated School of Clinical Medicine, Kunming Medical University, Kunming, Yunnan, China. ${ }^{3}$ Department of Chronic Disease Epidemiology, Yale School of Public Health Yale University, New Haven, CT, USA.

Received: 5 June 2019 Accepted: 12 December 2019 Published online: 23 December 2019

\section{References}

1. Hu X, Cook S, Salazar MA. Internal migration and health in China. Lancet. 2008;372:1717-9.

2. Gao Y, Li LP, Kim JH, Congdon N, Lau J, Griffiths S. The impact of parental migration on health status and health behaviours among left behind adolescents school children in China. BMC Public Health. 2010;10:56.

3. All-China Women's Federation. Research report on the situation of leftbehind children in rural China (excerpt). Chinese Women's Movement. 2008; 6:35-7. (In Chinese).

4. All-China Women's Federation. A research report on the situation of rural left-behind children and migrant children in China. Chinese Women's Movement. 2013;6:30-4. (In Chinese).

5. Liang Y, Wang L, Rui G. Depression among left-behind children in China. J Health Psychol. 2017;22:1897-905.

6. Dai Q, Chu RX. Anxiety, happiness and self-esteem of western Chinese leftbehind children. Child Abuse Negl. 2018;86:403-13.

7. Wang $Y$, Zhang $M$, Chen $H$. Self-injury among left-behind adolescents in rural China: the role of parental migration and parent-child attachment. Front Psychol. 2019;7:2672.

8. Nock NK, Joiner TE, Gordon KH, Lloyd-Richardson E, Prinstein MJ. Nonsuicidal self-injury among adolescents: diagnostic correlates and relation to suicide attempts. Psychiatry Res. 2016;144:65-72.

9. Fliege $H$, Lee JR, Grimm A, Klapp BF. Risk factors and correlates of deliberate self-harm behavior: a systematic review. J Psychosom Res. 2009;56:617-26.

10. Xin X, Wang Y, Fang J, Ming Q, Yao S. Prevalence and correlates of direct self-injurious behavior among Chinese adolescents: findings from a multicenter and multistage survey. J Abnorm Child Psychol. 2017;45:815-26.

11. Sourander A, Aromaa M, Pihlakoski L, Haavisto A, Rautava P, Helenius H, et al. Early predictors of deliberate self-harm among adolescents. A prospective follow-up study from age 3 to age 15. J Affect Disord. 2006;93:87-96.
12. Xu Y, Ma L. A discussion on characteristics and causes of self-harm in rural leftbehind teenagers: based on the survey in Macheng, Hubei province. J South Central Univ for Nationalities (Humanit Soc Sci Ed). 2013;33:90-6. (In Chinese).

13. Kong $X$, Liu Y, Zhao Q, Yuan L, Luo L. A study on the association between feeling of separation and self-harm in rural left-behind junior school students. Sci Technol West China. 2014;13:124-5. (In Chinese)

14. Foster T, Gillespie K, McClellad R. Mental disorders and suicide in Northern Ireland. Br J Psychiatry. 1997;170:447-52.

15. Whitlock J, Muehlenkamp J, Eckenrode J, Purington A, Baral Abrams G, Barreira $P$, et al. Nonsuicidal self-injury as a gateway to suicide in young adults. J Adolescents Health. 2013;52:486-92.

16. Chang H, Yan Q, Tang L, Huang J, Ma Y, Ye X, et al. A comparative analysis of suicide attempts in left-behind children and non-left-behind children in rural China. PLoS One. 2017;12:e0178743.

17. Luthar SS. Resilience and vulnerability: adaptation in the context of childhood adversities: Cambridge University Press; 2003.

18. Dong B, Yu D, Ren Q, Zhao D, Li J, Sun YH. The resilience status of Chinese left-behind children in rural areas: a meta-analysis. Psychol Health Med. 2019;24:1-13.

19. Huang L, Mossige S. Resilience in young people living with violence and self-harm: evidence from a Norwegian national youth survey. Psychol Res Behav Manag. 2015:8:231-8.

20. Dray J, Bowman J, Freund M, Campbell E, Wolfenden L, Hodder RK, et al. Improving adolescent mental health and resilience through a resiliencebased intervention in schools: study protocol for a randomised controlled trial. Trials. 2014;15:289.

21. Feng $Y$. The relation of adolescents' self-harm behaviors, Individual Emotion Characteristics and Family Environment Factors. Central China Norm Univ. 2008. (In Chinese).

22. Hu Y, Gan Y. Development and psychometric validity of the resilience scale for Chinese adolescents. Acta Psychol Sin. 2008;40:902-12. (In Chinese)

23. Greydanus DE, Shek D. Deliberate self-harm and suicide in adolescents. Keio J Med. 2009;58:144-51

24. Wang Y, Zhu X, Yi J, Yao S, Cai L, Bai M, et al. Self-harm and suicide behaviors in middle school students. Chin J Clin Psychol. 2012;20:666-8. (In Chinese)

25. Portzky G, De Wilde EJ, Van Heeringen K. Deliberate self-harm in young people: differences in prevalence and risk factors between the Netherlands and Belgium. Eur Child Adolesc Psychiatry. 2008;17:179-86.

26. Ma Y. Investigation of non-suicidal self-injury and influencing factors among rural middle school students. Huazhong Technological Uni. 2016; (In Chinese)

27. Yang $X$, Cui Z, Zhao J, Zhao J. The relationship between psychological and resilience, psychopathology and suicidal risks in Chinese college students. Chin J Health Psychol. 2013;9:1408-13. (In Chinese)

28. Haase JE. The adolescents resilience model as a guide to interventions. J Pediatr Oncol Nurs. 2004;21:289-99.

29. Nock MK, Prinstein MJ. A functional approach to the assessment of selfmutilative behavior. J Consult Clin Psychol. 2004;72:885-90.

30. Peng L, Zhang J, Li M, Li P, Zhang Y, Zuo X, et al. Negative life events and mental health of Chinese medical students: the effect of resilience, personality and social support. Psychiatry Res. 2012;196:138-41.

31. Hawton K, Saunders KE, O'Connor RC. Self-harm and suicide in adolescents. Lancet. 2012;379:2373-82.

32. Schafer JO, Naumann E, Holmes EA, Tuschen-Caffier B, Samson AC. Emotion regulation strategies in depressive and anxiety symptoms in youth: a metaanalytic review. J Youth Adolesc. 2017;46:261-76.

33. McLaughlin KA, Hatzenbuehler ML, Mennin DS, Nolen-Hoeksema S. Emotion dysregulation and adolescent psychopathology: a prospective study. Behav Res and Ther. 2011:49:544-54.

34. Zubrick SR, Hafekost J, Johnson SE, Sawyer MG, Patton G, Lawrence D. The continuity and duration of depression and its relationship to non-suicidal self-harm and suicidal ideation and behavior in adolescents. J Affect Disord. 2017;220:49-56.

35. Viana AG, Dixon LJ, Berenz EC, Espil FM. Trauma and deliberate self-harm among inpatient adolescents: the moderating role of anxiety sensitivity. Psychol Trauma. 2017;9:509-17.

36. Messer JM, Fremouw WJ. A critical review of explanatory models for selfmutilating behaviors in adolescentss. Clin Psychol Rev. 2008;28:162-78.

37. Xu Z, Su H, Wu J, Chang W, Sun Y. Self-injurious behavior among rural stayat-home middle school students and its relationship with internal and external locus of control. Chin J Public Health. 2010;26:868-9. (In Chinese) 
38. Wan Y, Chen J, Sun Y, Hu C, Tao F. Relationship between parent-child attachment and deliberate self-harm behaviors in middle school students, Chin J Child Health Care. 2013;21:1239-42. (In Chinese)

39. Claes L, Raedt RD, Walle MVD, Bosmans G. Attentional bias moderates the link between attachment-related expectations and nonsuicidal self-injury. Cogn Ther Res. 2016;40:540-8.

40. Ai H, Hu J. Psychological resilience moderates the impact of social support on loneliness of "left-behind" children. J Health Psychol. 2014;21:1066-73.

41. Guo J, Ren X, Wang X, Qu Z, Zhou Q, Ran C, et al. Depression among migrant and left-behind children in China in relation to the quality of parent-child and teacher-child relationships. PLoS One. 2015;10:e0145606.

42. Wu YL, Zhao X, Ding XX, Yang HY, Qian ZZ, Feng F, et al. A prospective study of psychological resilience and depression among left-behind children in China. J Health Psychol. 2017;22:627-36.

43. Dray J, Bowman J, Campbell E, Freund M, Hodder R, Wolfenden L, et al. Effectiveness of a pragmatic school-based universal intervention targeting student resilience protective factors in reducing mental health problems in adolescents. J Adolesc. 2017;57:74-89.

44. Li J. Intervention of group counseling based on emotion management in adolescent non-suicidal self-injury. Hunan Norm Univ. 2016; (In Chinese)

45. Su S, Li X, Lin D, Xu X, Zhu M. Psychological adjustment among left-behind children in rural China: the role of parental migration and parent-child communication. Child Care Health Dev. 2013;39:162-70.

46. Wang L, Feng Z, Yang G, Yang Y, Dai Q, Hu C, et al. The epidemiological characteristics of depressive symptoms in the left-behind children and adolescents of Chongqing in China. J Affect Disord. 2015;177:36-41.

\section{Publisher's Note}

Springer Nature remains neutral with regard to jurisdictional claims in published maps and institutional affiliations.

Ready to submit your research? Choose BMC and benefit from:

- fast, convenient online submission

- thorough peer review by experienced researchers in your field

- rapid publication on acceptance

- support for research data, including large and complex data types

- gold Open Access which fosters wider collaboration and increased citations

- maximum visibility for your research: over $100 \mathrm{M}$ website views per year

At $\mathrm{BMC}$, research is always in progress.

Learn more biomedcentral.com/submissions 